\title{
Japon. La politique éducative depuis les années
} 1990

Études de cas

\section{Sachiko Fujii et Daisuke Sonoyama}

\section{OpenEdition}

\section{Journals}

Édition électronique

URL : https://journals.openedition.org/ries/1261

DOI : 10.4000/ries. 1261

ISSN : 2261-4265

\section{Éditeur}

France Education international

Édition imprimée

Date de publication : 1 décembre 2005

Pagination : 48-50

ISSN : 1254-4590

Référence électronique

Sachiko Fujii et Daisuke Sonoyama, « Japon. La politique éducative depuis les années 1990 », Revue internationale d'éducation de Sèvres [En ligne], 40 | décembre 2005, mis en ligne le 17 novembre 2011, consulté le 06 juillet 2021. URL : http://journals.openedition.org/ries/1261 ; DOI : https://doi.org/ 10.4000/ries. 1261

Ce document a été généré automatiquement le 6 juillet 2021.

(c) Tous droits réservés 


\title{
Japon. La politique éducative depuis les années 1990
}

Études de cas

\author{
Sachiko Fujii et Daisuke Sonoyama
}

1 La politique éducative du Japon a été l'objet d'une profonde mutation dans les années 1990. L'idée dominante est l'individualisation de l'enseignement et les réformes tendant à un enseignement "répondant à l'individualité de l'élève » ou encore « répondant aux besoins de l'élève » se poursuivent encore aujourd'hui. Cette tendance politique tire son origine du Conseil extraordinaire sur l'Éducation, Rinkyôshi, instauré en 1984 sous le contrôle direct du Premier ministre. Rinkyôshi, considérant que «l'uniformité, la rigidité, et la fermeture» étaient les maladies profondes de l'enseignement public du pays, a affirmé qu'il était nécessaire de les supprimer et d'établir le "principe de la prépondérance de l'individualité », à savoir « le principe du respect de l'individu, le respect de la personnalité, la liberté et l'autonomie, la responsabilité individuelle». Et comme moyen essentiel pour atteindre ce but, elle a lancé la « libéralisation de l'enseignement ». Autrement dit, on est allé dans le sens du renforcement de l'autonomie des établissements scolaires et des comités municipaux de l'instruction publique, et donc d'un affermissement, en matière d'éducation, du principe de la liberté, de l'autonomie, et de la responsabilité individuelle, ainsi que de la promotion d'une plus grande variété des options possibles.

\section{L'épanouissement de l'individu}

2 La nouvelle politique éducative est clairement exposée dans les nouvelles Directives d'enseignement de 1989 qui stipulent que les aptitudes scolaires à favoriser sont l'envie d'étudier par soi-même, la force de réflexion, la capacité de jugement et d'expression, tentant ainsi de passer d'une façon unilatérale d'enseigner, en usage jusqu'alors, à un apprentissage centré sur l'élève. En même temps, elle pose comme base de l'évaluation "les caractéristiques de l'apprentissage avec points de vue différenciés", caractéristiques évaluées subjectivement par les enseignants, telles que «l'intérêt, la 
volonté, le comportement ", ou encore « la réflexion, le jugement », et cela pour chaque matière. Enfin, elle attribue à la notation - celle, ordinaire, qui consiste à évaluer le niveau scolaire par des tests écrits - le statut de complément. Le Mombushô (ministère de l'Éducation et de la Culture) a appelé cette nouvelle méthode d'évaluation du niveau scolaire la «nouvelle observation du niveau scolaire». Ensuite, on a fait progresser la diversité et l'originalité de l'enseignement dans les lycées ("filière polyvalente", création de lycées d'un nouveau type), la diversité des examens d'entrée dans les lycées et les universités, etc. Et le rapport du Conseil central de l'éducation (Chukyôshin) de juillet 1996, proposant de poser dorénavant comme objectif d'éducation le "développement de la capacité de vivre, dans un cadre non-contraignant ", a défendu la thèse d'un changement faisant passer d'une éducation qui tendait à l'acquisition de connaissances à une éducation développant la «force de vivre » (ikiru chikara), c'est-àdire la capacité d'étudier et de réfléchir par soi-même.

3 Ainsi, on a commencé à aller vers une éducation qui s'appuyait étroitement sur les nouvelles idées libérales selon lesquelles il est plus important de répondre aux divers besoins personnels et collectifs que de rechercher la rationalité sociale.

\section{La confrontation à l'économie de marché}

La politique éducative néo-libérale apparaît clairement, surtout après 1997, et on peut y remarquer plusieurs caractéristiques propres au Japon. Déjà, dans les années quatrevingts, de puissantes réformes néo-libérales de l'éducation avaient été mises en place aux États-Unis et en Angleterre. Elles visaient à rehausser le niveau scolaire et à l'excellence en éducation par l'introduction d'un système efficace et compétitif. On voit là clairement une structure qui tend à l'élévation du niveau scolaire par la concurrence et la loi du marché, sur la base de la liberté et de la responsabilité de l'individu, pour la reconstitution de la compétitivité de l'industrie du pays. Mais la compétitivité n'était pas un thème porteur dans le Japon des années quatre-vingt-dix. Il s'agissait plutôt, pour sortir de la situation de contrôle fort et d'éducation "bourrage de crâne ", de mettre l'accent sur l'originalité et la liberté dans l'éducation, les points importants étant l'assouplissement du système et aussi le développement de la personnalité et de la liberté, afin de freiner l'esprit de compétition.

5 Ainsi, de concert avec le courant de pensée néo-libéral et la dérégulation qui ont prévalu dans le domaine de l'économie politique à partir de 1993, une éducation ayant pour but la formation de la «force de vivre » dans un cadre non-contraignant, devient l'idée de base. Cette situation où l'on ne visait pas, dans les lieux d'enseignement, la réalisation d'un objectif quantitatif fixé, mais plutôt l'épanouissement de l'individu s'est poursuivie dans les années quatre-vingt-dix.

6 Enfin, le Japon est actuellement dans une époque de "tests d'évaluation du niveau scolaire » et de compétition. Du fait qu'on tente de faire de l'enseignement scolaire un pseudo-marché, chaque école est interrogée sur ses résultats et doit désormais en assumer la responsabilité. C'est l'avènement d'une époque sévère tant pour les écoles que pour les enseignants. Diverses critiques se sont bien sûr élevées. Cela ne ferait qu'accroître la liberté d'éducation d'une partie des parents, cela amplifierait les écarts de niveau entre les écoles, cela gâcherait de fait les chances d'éducation des classes sociales les moins élevées, et cela accentuerait la fracture sociale. 
7 Depuis Rinkyôshin, la politique éducative ne parvient pas à jouer un rôle actif de guide. L'éducation suit le mouvement des mécanismes de l'économie de marché. En arrièreplan, il y a la réalité des écoles japonaises, surtout publiques, qui doivent faire face à plusieurs problèmes et notamment à celui du rejet des études par les élèves.

Comment retrouver une éducation de haute qualité ? Si tel est l'objectif le plus important attendu de la politique éducative actuelle, alors sur quelle conception et quelle stratégie doit-il reposer ? Comment faire face aux conséquences négatives qui résultent de l'extension des possibilités de choix et de la multiplicité des établissements fournisseurs de services éducatifs ? Le nombre de points à débattre est infini. Jusqu'à présent, le Monkashô (ministère de l'Éducation et des Sciences) s'est totalement abstenu d'une évaluation de la politique éducative actuelle.

INDEX

Index géographique : Japon

\section{AUTEURS}

\section{SACHIKO FUJII}

Professeur, université d'Utsonomiya.

\section{DAISUKE SONOYAMA}

Professeur, université d'Oïta. 